\title{
Surgical glove perforation during laparoscopic colorectal procedures
}

\author{
Shinsei Matsuoka ${ }^{1} \cdot$ Takayuki Kondo $^{2} \cdot$ Ryo Seishima $^{1} \cdot$ Koji Okabayashi $^{1} \cdot$ Masashi Tsuruta $^{3} \cdot$ Kohei Shigeta $^{1}$. \\ Takashi Ishida $^{3} \cdot$ Hirotoshi Hasegawa $^{4} \cdot$ Yuko Kitagawa ${ }^{1}$
}

Received: 18 November 2020 / Accepted: 29 July 2021 / Published online: 11 August 2021

(c) The Author(s), under exclusive licence to Springer Science+Business Media, LLC, part of Springer Nature 2021

\begin{abstract}
Background It has been reported that in conventional open surgery, approximately $10 \%$ of surgical gloves are perforated during surgery without being noticed. To protect both the patient and medical staff from harm, double gloving or changing gloves routinely at certain intervals during surgery is recommended. However, whether these protective measures are also necessary for laparoscopic colorectal surgery is unknown because the actual perforation rate during laparoscopic procedures is unclear.

Methods Seventy-seven laparoscopic colorectal surgeries were evaluated, and a total of 616 surgical gloves used in the surgeries were collected for analysis. The presence of glove perforation was tested by the standard water-leak test method (EN455-1).

Results Seven perforations were detected (1.1\%). The duration of the laparoscopic procedure was not a statistically significant risk factor for glove perforation $(p=0.41)$. Postoperative surgical site infections (SSIs) were observed in 12 cases $(15.6 \%)$, but there was no significant correlation between the presence of glove perforation and SSI $(p=0.92)$. According to the bacterial cultivation results, the majority of causative agents of SSI were enterobacteria, which belong to the major gut flora.

Conclusion Although the perforation rate was considerably lower than that in open surgery, surgical glove perforation occurred during laparoscopic procedures. Double gloving in laparoscopic colorectal surgery is recommended not to prevent SSI but to protect medical workers from harmful infections after direct contact with the patient.
\end{abstract}

Keywords Surgical glove perforation $\cdot$ Laparoscopic colorectal surgery $\cdot$ Surgical site infection

The appropriate wearing of surgical gloves is the most important protective measure against the transmission of infectious agents from a patient to the surgical staff. Nevertheless, perforation of surgical gloves occurs very frequently in clinical practice. According to past reports, approximately $10 \%$ of surgical gloves are perforated during surgery [1-3].

Shinsei Matsuoka and Takayuki Kondo contributed equally to this work.

Koji Okabayashi

okabayashikoji@gmail.com

1 Department of Surgery, Keio University School of Medicine, 35 Shinano-machi, Shinjuku-ku, Tokyo 160-8582, Japan

2 Department of Surgery, Kawasaki Municipal Hospital, Kawasaki, Japan

3 Department of Surgery, Faculty of Medicine, International University of Health and Welfare, Narita, Japan

4 Department of Surgery, Tokyo Dental College Ichikawa General Hospital, Ichikawa, Japan
Furthermore, approximately $70 \%$ of surgical glove perforations go unnoticed during surgical procedures [3]. These data suggest that surgical staff are potentially unknowingly exposed to infectious agents, which is dangerous for medical workers. In addition, glove perforation has the potential to cause surgical site infection (SSI) by transmitting bacteria to the patient's body, which is dangerous for patients [4]. As colorectal surgery is generally associated with a higher SSI rate than other types of surgeries, the prevention of glove perforation during colorectal surgery may have a greater impact on decreasing the SSI rate than other protection measures [5-7]. To avoid breaching the protective barrier, thereby protecting both the medical workers and the patients from harm, double gloving or changing gloves at routine intervals during surgery is recommended $[3,8,9]$.

Colorectal surgery has rapidly shifted from conventional open surgery to laparoscopic surgery in recent decades. Although reports regarding glove perforation involve mostly open surgery, it has been recently reported that the 
perforation rate in laparoscopic surgery is lower than that in conventional open surgery [10]. As laparoscopic surgery generally does not require manual sutures and involves relatively small skin incisions and the absence of surgical manoeuvres with the hands, it is highly likely to be protective against glove perforation. Therefore, we hypothesized that double gloving or changing surgical gloves at a certain interval may be unnecessary during laparoscopic procedures for colorectal surgery. Based on this hypothesis, medical staff may be able to reduce the frequency of glove changes in laparoscopic surgery. In general, laparoscopic surgery tends to take longer than open surgery in the same area [11]. Therefore, if routine surgical glove changes at certain intervals are performed similarly to those in open surgery, unfavourable economic problems will arise by increasing the consumption of surgical gloves. Avoiding excessive surgical glove use may contribute to saving medical resources without decreasing safety.

The objectives of this study were to examine the prevalence of glove perforation during laparoscopic colorectal procedures and to describe any relationship between glove perforation and SSIs. These will help to assess the necessity of routine surgical glove changes during laparoscopic procedures.

\section{Materials and methods}

This is a prospective observational single-institutional study performed at Keio University Hospital to investigate actual perforation rate during laparoscopic colorectal procedures to examine the prevalence of glove perforation in laparoscopic procedures.

\section{Participants}

Seventy-seven patients who performed laparoscopic colorectal surgeries between August 2015 and March 2019 were enrolled in this study. All surgeries were scheduled and performed by four surgical members: an operator, first assistant, second assistant, and scrub nurse. All of operators were colorectal surgery specialists. The first assistants and the second assistants were general surgery specialists or trainees. This study was approved by the institutional ethics committee and completed written informed consent from patient was obtained.

\section{Procedures}

We aimed to evaluate surgical glove perforation during "pure" laparoscopic procedures (Fig. 1). To do so, the gloves of all surgical members were changed following the initiation of pneumoperitoneum and the establishment of the trocars. During the laparoscopic procedure, the same gloves were worn unless apparent holes were detected. At the time of pneumoperitoneum termination, all gloves from all surgical members were collected. Accordingly, extra-abdominal procedures such as skin incision, intestine resection, anastomosis, and wound closure were excluded from the evaluation. The presence of glove perforation was tested by the standard water-leak test method (EN455-1) [12]. In brief, the gloves were filled with $1000 \mathrm{ml}$ of water and observed to detect any holes. Protexis ${ }^{\mathrm{TM}}$ latex micro surgical gloves (Cardinal Health, Ohio, USA) were used in all surgeries. All members of the surgical team in this study were single-gloved during surgery. Detailed information regarding the surgeries (operative procedure, primary disease, glovewearing time, blood loss) as well as patient information (age, sex, body mass index), which were considered potential confounders or predictors, were collected. The records of SSI, including superficial incisional SSI, deep incisional SSI, and organ/space SSI, were collected. Bacterial cultivation was performed as soon as any infectious symptoms appeared, and the results were recorded.

\section{Outcomes of interest}

The primary outcome was the occurrence of surgical glove perforation. Detailed information regarding perforations (i.e. which members and which areas of the gloves were perforated) were collected. The secondary outcome was
Fig. 1 Scheme of the study. During the "pure" laparoscopic procedure, the same gloves were used until the pneumoperitoneum procedure was terminated

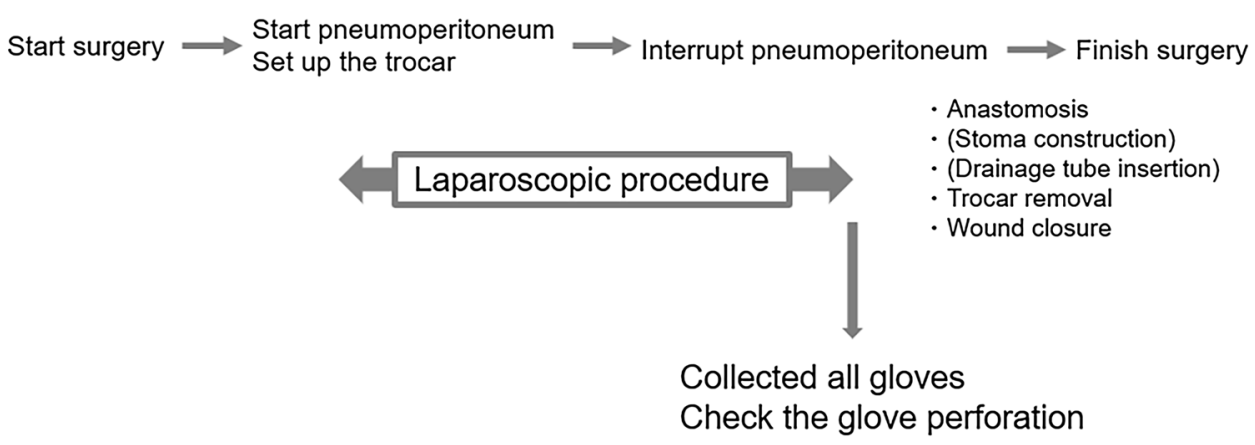


the occurrence of SSI and the correlation between glove perforation and SSI.

The predictor of glove perforation is duration of laparoscopic procedures. Potential confounders such as operative procedures and experience of operator were collected.

\section{Statistical methods}

All statistical analyses were performed with the software package STATA/IC (STATA Corp., 2019. Stata Statistical Software: Release-16. StataCorp LP, College Station, TX, USA). In the descriptive analysis, continuous variables are described as means with standard deviations or medians with maximum and minimum ranges, whereas categorical variables are described as frequencies. Chi-square test and Mann-Whitney U test were used for comparison of variables as appropriate.

Table 1 Patient characteristics

\begin{tabular}{ll}
\hline Age (years) & $68.8 \pm 14.9^{*}$ \\
Sex (male/female) & $48 / 29$ \\
Body mass index $\left(\mathrm{kg} / \mathrm{m}^{2}\right)$ & $22.6 \pm 3.9^{*}$ \\
Primary disease $($ cases $)$ & \\
Colorectal cancer & 75 \\
Crohn's disease & 1 \\
Others & 1 \\
Operative procedure (cases) & \\
Ileocecal resection & 17 \\
Right hemicolectomy & 12 \\
Left hemicolectomy & 5 \\
Anterior resection & 24 \\
Partial resection & 13 \\
Others & 6 \\
Blood loss (g) & $36.9 \pm 72.4^{*}$ \\
\hline
\end{tabular}

${ }^{*}$ Mean \pm SD

\section{Results}

\section{Patient characteristics}

The characteristics of the 77 patients are shown in Table 1. Most of the surgeries were performed for colorectal cancer treatment ( 74 cases of colorectal cancer, 1 case of Crohn's disease, 2 cases of other diseases). Laparoscopic ileocecal resection was performed in 17 cases, laparoscopic right hemicolectomy in 12 cases, laparoscopic left hemicolectomy in 5 cases, laparoscopic anterior resection of the rectum in 24 cases, and laparoscopic partial colonic resection in 13 cases.

\section{Glove perforation}

A total of 616 surgical gloves used in the surgeries were collected for analysis. Amongst them, 7 perforations $(1.1 \%)$ were detected by the water-leak test: 1 in an operator's glove, 2 in first assistants' gloves, 2 in second assistants' gloves, and 2 in scrub nurses' gloves (Table 2; Fig. 2). In one case of first assistant glove perforation, data regarding the perforation site were missing. Amongst

\section{O Operator \\ $1^{\text {st }} 1^{\text {st }}$ assistant \\ $2^{\text {nd }} 2^{\text {nd }}$ assistant \\ $S$ Scrub nurse}

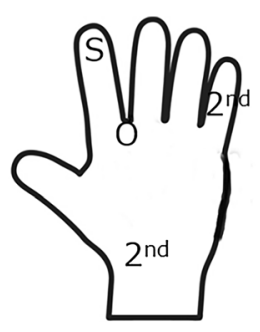

L

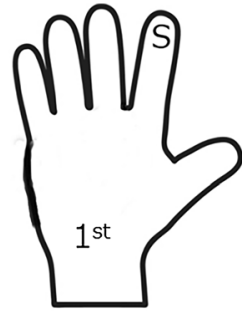

$\mathrm{R}$
Fig. 2 A schematic summary of glove perforation sites. Each symbol representing the surgical member indicated the perforated sites on gloves. $L$ left, $R$ right
Table 2 Summary of surgical glove perforation cases

\begin{tabular}{llll}
\hline Case & Operative procedure & $\begin{array}{l}\text { Glove wear time } \\
(\mathrm{min})\end{array}$ & Surgical member with glove perforation \\
\hline 1 & Sigmoidectomy & 141 & Left little finger of 2nd assistant \\
2 & Abdominoperineal resection & 173 & Left index finger of scrub nurse \\
3 & Ileocecal resection & 200 & $1^{\text {st }}$ assistant's hand $*$ \\
4 & Anterior resection & 219 & Right palm of 1st assistant \\
5 & Right hemicolectomy & 278 & Right index finger of scrub nurse \\
6 & Anterior resection & 303 & Left palm of 2nd assistant \\
7 & Anterior resection & 477 & Interdigital space between operator's \\
& & & left index finger and middle finger \\
\hline
\end{tabular}

*Data on the perforation site were missing 
all the perforations, only two perforations $(28.6 \%)$ were noticed by the staff member during the laparoscopic procedure.

The overall median duration of the laparoscopic procedure was $205 \mathrm{~min}$ (93-493 $\mathrm{min}$ ). The median time in the cases with perforated gloves was 219 min (141-477 min), whereas that in cases with non-perforated was $204 \mathrm{~min}$ (93-493 min) (Fig. 3). There was no significant correlation between glove perforation and laparoscopic procedure duration time $(p=0.41)$. No significant difference of glove perforation rate was identified between colon and rectum surgery (colon surgery $6.4 \%$ vs rectal surgery $14.8 \%, p=0.23$ ).

\section{Postoperative SSI}

Postoperative SSIs occurred in 12 cases (15.6\%); one case amongst the 7 glove perforation cases (14.3\%) and 11 cases amongst the 70 non-perforation cases (15.7\%) occurred. There was no significant correlation between the presence of glove perforation and SSI $(p=0.92)$.

We next focused on the bacterial cultivation survey results at SSI sites to assess the impact of skin flora, which could be imported from medical workers to the patient body by glove perforation. According to the bacterial cultivation results, the majority of the causative agents of SSI were enterobacteria (Table 3 ). In the case in which glove perforation was detected on the operator's left hand, Pseudomonas aeruginosa and Enterobacter cloacae were detected. Staphylococcus aureus, a species that is part of the natural skin flora, was detected in a case with no glove perforation. Bacterial cultivation was not performed in one non-perforation case at the doctor's discretion.

Fig. 3 A histogram showing the correlation between glove perforation and laparoscopic procedure duration time

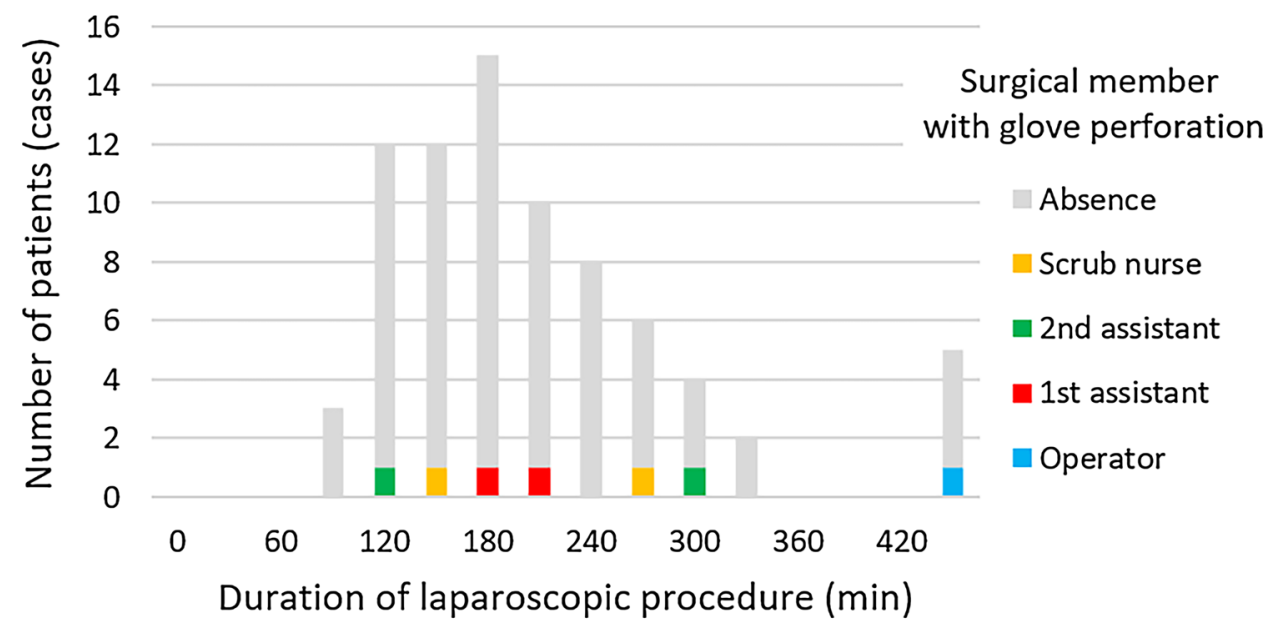

Table 3 Summary of the SSI cases

\begin{tabular}{llc}
\hline Case & Detected bacteria & $\begin{array}{c}\text { Presence of } \\
\text { glove perfora- } \\
\text { tion }\end{array}$ \\
\hline 1 & & Yes \\
2 & Pseudomonas aeruginosa, Enterobacter cloacae & No \\
3 & Escherichia coli, Enterobacter, Enterococcus, Bacteroides & No \\
4 & Pseudomonas aeruginosa, Escherichia coli, Enterococcus faecalis, Bacteroides fragilis & No \\
5 & Pseudomonas aeruginosa, Bacteroides thetaiotaomicron & No \\
6 & Bacteroides vulgatus & No \\
7 & Pseudomonas aeruginosa, Enterococcus faecalis & No \\
8 & Staphylococcus aureus & No \\
9 & Enterobacter cloacae, Enterococcus faecalis, Bacteroides fragilis & No \\
10 & Enterococcus casseliflavus & No \\
11 & Enterobacter cloacae, Klebsiella pneumoniae, Enterococcus sp., Candida glabrata & No \\
12 & Nocardia sp. & No
\end{tabular}




\section{Discussion}

We revealed that glove perforation occurred even during laparoscopic procedures. The actual perforation rate was $1.1 \%$ in this study; this rate was significantly lower than that in open surgery according to previous reports [1-3]. Intriguingly, there was no significant difference in the glove perforation rate amongst surgical members. In a previous report evaluating open surgery, the operator had the greatest risk of glove perforation [13]. The possible reason for this inconsistency could be that surgical members' hand, especially those of operator, have less opportunity to come close to surgical instruments such as scissors, clamps, and electrocautery devices in laparoscopic procedures. Although a prolonged laparoscopic procedure duration usually reflects surgical challenges, such as haemorrhage, adhesion, or visceral obesity, duration of laparoscopic procedures was not significantly associated with glove perforation. In open surgery, a prolonged duration of surgical glove wear is reported to be a risk factor for glove perforation [14]. Therefore, changing gloves during surgery is recommended; the appropriate glove change interval is reported to be between 90 and 150 min [14-16]. However, in laparoscopic procedures, the risk of surgical glove perforation may not be increased by extending the operation time. The main motion performed by both the operator and assistants during the laparoscopic procedure is grasping, which may not damage the gloves and thus allow prolonged use. These results imply that glove changing at certain intervals may not be necessary during laparoscopic procedures.

The correlation between glove perforation and SSI was unclear in our study. Regardless of the status of glove perforation, enterobacteria were present in most of the SSI cases. In previous literature on SSI after colorectal surgery, almost all microorganisms isolated from SSI sites were enterobacteria [17]. Based on the hypothesis that glove perforation may cause SSI, skin flora should be detected at SSI sites. However, the frequent detection of gut flora but not skin flora in this study indicates that SSI in laparoscopic surgery is mostly caused by the inappropriate handling of intestines, resulting in patient exposure to gastrointestinal content. Thus, the main risk of glove perforation in this study was the opportunity for the transmission of infectious agents from patients to surgical members and not SSI.

In summary, according to our results, to minimize harm from glove perforation, double gloving might be a better practice than glove changing. Although there are no data regarding the infection rate of surgical team members after glove perforation, double gloving has been shown to offer greater protection against visible skin contamination than single gloving [18]. Amongst the methods of double gloving, we recommend double indicator gloving, which enables medical staff to visualize perforations in the outer gloves by colour changes. This practice is reported to have a better outer glove perforation detection rate than conventional double gloving in orthopaedic and trauma surgeries [19]. It is also considered to have advantages in laparoscopic colorectal surgery because quickly changing the perforated outer glove is necessary to avoid transmitting infectious agents from the patient to the surgical member. Furthermore, standardization of double indicator gloving amongst the surgical team may become a protective measure during the epidemic phases of emerging infectious diseases such as COVID-19. Some surgeons and surgical nurses may feel uncomfortable with double gloving because it may weaken their sense of touch. Further investigation of operative team members' satisfaction with double gloving is needed.

The issue of defective products cannot be ignored in this study. Ersozlu et al. reported that $1 \%$ of unused surgical gloves had holes [20]. According to the manufacturer's product information, the acceptable quality limit (AQL) of the gloves used in this study is 0.65 , which means that a maximum of $0.65 \%$ of unused gloves may have holes. Before starting this study, we estimated that the frequency of defective product would be much lower than the perforation rate during surgery. However, unexpectedly, the actual perforation rate was too low (1.1\%) to ignore the frequency of product defects. In other words, laparoscopic procedures may not increase the perforation rate beyond that arising from defective products; the rate of increase was only $0.45 \%$. As surgical members cannot do anything about defective products, this is another reason for recommending double gloving.

There are some limitations in this study: First, this is an observational study, and the sample size is too small to allow a definitive conclusion to be reached. A larger prospective study to validate our results is expected in the future. Second, the surgical members participating in this study varied in terms of their experience levels. The proficiency level of each member may influence the glove perforation rate because trainees sometimes tend to hold and use devices in inappropriate ways. Third, we did not have any information about the surgical devices. The use of some fine devices for laparoscopic procedures, which may cause perforation more frequently than conventional devices, could influence the results.

In conclusion, surgical glove perforation can occur even during "pure" colorectal laparoscopic procedures. In addition, it is important to note that all surgical members have equal risks of glove perforation, which may expose them to infectious agents. Double indicator gloving could be a reasonable technique to reduce harm due to glove perforation. However, the incidence of perforation during laparoscopic 
surgery was much lower than that during conventional open surgery, suggesting an additional benefit of laparoscopic surgery.

\section{Declarations}

Disclosures Dr. Shinsei Matsuoka, Dr. Takayuki Kondo, Dr. Ryo Seishima, Dr. Koji Okabayashi, Dr. Masashi Tsuruta, Dr. Kohei Shigeta, Prof. Hirotoshi Hasegawa, and Prof. Yuko Kitagawa have no conflicts of interest or financial ties to disclose.

\section{References}

1. Greco RJ, Wheatley M, McKenna P (1993) Risk of blood contact through surgical gloves in aesthetic procedures. Aesthetic Plast Surg 17(2):167-168

2. Dodds RD, Guy PJ, Peacock AM, Duffy SR, Barker SG, Thomas MH (1988) Surgical glove perforation. Br J Surg 75(10):966-968

3. Laine T, Aarnio P (2001) How often does glove perforation occur in surgery? Comparison between single gloves and a double-gloving system. Am J Surg 181(6):564-566

4. Misteli H, Weber WP, Reck S, Rosenthal R, Zwahlen M, Fueglistaler P, Bolli MK, Oertli D, Widmer AF, Marti WR (2009) Surgical glove perforation and the risk of surgical site infection. Arch Surg 144(6):553-558

5. Itani KM, Wilson SE, Awad SS, Jensen EH, Finn TS, Abramson MA (2006) Ertapenem versus cefotetan prophylaxis in elective colorectal surgery. N Engl J Med. https://doi.org/10.1056/NEJMo a054408Dec21

6. Tang R, Chen HH, Wang YL, Changchien CR, Chen JS, Hsu KC, Chiang JM, Wang JY (2001) Risk factors for surgical site infection after elective resection of the colon and rectum: a singlecenter prospective study of 2,809 consecutive patients. Ann Surg 234(2):181-189

7. Anthony T, Murray BW, Sum-Ping JT, Lenkovsky F, Vornik VD, Parker BJ, McFarlin JE, Hartless K, Huerta S (2011) Evaluating an evidence-based bundle for preventing surgical site infection: a randomized trial. Arch Surg 146(3):263-269

8. Quebbeman EJ, Telford GL, Wadsworth K, Hubbard S, Goodman H, Gottlieb MS (1992) Double gloving. Protecting surgeons from blood contamination in the operating room. Arch Surg 127(2):213-216

9. Chittacharoen P, Chittacharoen A, Ayudhya NI (2004) Randomized controlled trial of glove perforation in single- and double-gloving in episiotomy repair after vaginal delivery. $\mathbf{J}$ Obstet Gynaecol Res 30(5):354-357

10. Laine T, Kaipia A, Santavirta J, Aarnio P (2004) Glove perforations in open and laparoscopic abdominal surgery: the feasibility of double gloving. Scand J Surg 93(1):73-76

11. Fukuoka E, Matsuda T, Hasegawa H, Yamashita K, Arimoto A, Takiguchi G, Yamamoto M, Kanaji S, Oshikiri T, Nakamura T, Suzuki S, Kakeji Y (2019) Laparoscopic vs open surgery for colorectal cancer patients with high American Society of Anesthesiologists classes. Asian J Endosc Surg. https://doi.org/10.1111/ ases.12766,Dec18

12. European Committee for Standardization (2017) Info-EN 455 standards. http://www.en-medipart.com.my/index.php/2017/02/ 08/info-en-455-standards/. February 8, 2017.

13. Sayın S, Yilmaz E, Baydur H (2019) Rate of glove perforation in open abdominal surgery and the associated risk factors. Surg Infect 20(4):286-291

14. Harnoss JC, Partecke LI, Heidecke CD, Hübner NO, Kramer A, Assadian O (2010) Concentration of bacteria passing through puncture holes in surgical gloves. Am J Infect Control 38(2):154-158

15. Partecke LI, Goerdt AM, Langner I, Jaeger B, Assadian O, Heidecke CD, Kramer A, Huebner NO (2009) Incidence of microperforation for surgical gloves depends on duration of wear. Infect Control Hosp Epidemiol 30(5):409-414

16. de Oliveira AC, Gama CS (2014) Evaluation of surgical glove integrity during surgery in a Brazilian teaching hospital. Am J Infect Control 42(10):1093-1096

17. Kalakouti E, Simillis C, Pellino G, Mughal N, Warren O, Mills S, Tan E, Kontovounisios C, Tekkis PP (2017) Characteristics of surgical site infection following colorectal surgery in a tertiary center: extended-spectrum $\beta$-lactamase-producing bacteria culprits in disease. Wounds 30(4):108-113

18. Thomas S, Agarwal M, Mehta G (2001) Intraoperative glove perforation-single versus double gloving in protection against skin contamination. Postgrad Med J 77(909):458-460

19. Laine T, Aarnio P (2004) Glove perforation in orthopaedic and trauma surgery. A comparison between single, double indicator gloving and double gloving with two regular gloves. J Bone Jt Surg Br 86(6):898-900

20. Ersozlu S, Sahin O, Ozgur AF, Akkaya T, Tuncay C (2007) Glove punctures in major and minor orthopaedic surgery with double gloving. Acta Orthop Belg 73(6):760-764

Publisher's Note Springer Nature remains neutral with regard to jurisdictional claims in published maps and institutional affiliations. 